\section{Canadian Resident Research Projects and Peer-Reviewed Publication}

It was with interest that I read the paper by Hung and Duffett $^{1}$ reporting publication rates for pharmacy residents' research projects. Although I was pleased to find that the authors had cited my own paper ${ }^{2}$ reporting a similar project concerning the disposition of research projects by medical residents at the University of British Columbia, I was also saddened and disappointed to learn that the recent outcomes among pharmacy residents were essentially the same: a dismal conversion of residents' research projects into peer-reviewed publications.

Back in 2001, we discovered that the proportion of medical residents' research projects that were published in peer-reviewed journals was a paltry $28 \%$. In 2013, Hung and Duffett have reported that the overall publication rate for pharmacy residents' projects (between 1999/2000 and 2008/2009) was 32.2\%, with a rate of only $20.6 \%$ for full-text papers. As Hung and Duffett note, this situation appears to be generalized, as US pharmacy residents' publication rates ranged from $20 \%$ in 1981 to $12 \%$ in $2001 .^{3}$ Given the recent proliferation of new peer-reviewed and indexed journals, both in print and online, one might have expected to see a more robust publication rate for residents' projects.

The hard question that remains is "why"? One reason may be that many of the projects are simply not strong enough to merit publication, but it is difficult to believe that $70 \%$ to $80 \%$ of residents' projects are weak and unworthy. If that were true, then it would be appropriate to question the overall value of subjecting residents to the rigours of a research project: they might be better off studying something else. Both our group ${ }^{2}$ and the authors of the most recent paper ${ }^{1}$ theorized about the usual reasons: lack of time, lack of interest (on the part of either residents or faculty), etc. But perhaps what is really needed is a change in academic culture and attitude. The benefits of publishing should be emphasized: benefits to the individual (personal and professional satisfaction, enhanced professional profile), the department (enhanced academic stature), and the profession (improvements in clinical knowledge that may translate into better clinical practice). Having been a supervisor or co-supervisor for many residents' research projects, in both medicine/surgery and pharmacy, I realize that sometimes the immediate end point may appear to be a presentation at the local resident research day or national conference. The real (hard) end point, however, should be the submission of a manuscript to a peer-reviewed journal, with "winning" defined as publication of the submitted manuscript. To quote the late, revered professional football coach Vince Lombardi, "Winning isn't everything. . . it is the only thing." Perhaps residents and their faculty supervisors need to try to "win" more often.

\section{References}

1. Hung M, Duffett M. Canadian pharmacy practice residents' projects: publication rates and study characteristics. Can J Hosp Pharm. 2013;66(2):86-95.

2. Gill S, Levin A, Djurdjev O, Yoshida EM. Obstacles to residents' conducting research and predictors of publication. Acad Med. 2001;76(5):477.

3. McKelvey RP, Hatton RC, Kimberlin CA. Pharmacy resident project publication rates and study designs from 1981, 1991 and 2001. Am J Health Syst Pharm. 2010;67(10):830-6.
Eric M Yoshida, MD, MHSC, FRCPC, FACP, FACG, FCAHS

Division of Gastroenterology

University of British Columbia

Vancouver General Hospital

Vancouver, British Columbia

Competing interests: The author's institution has received unrestricted grants (or grants pending), for research on topics other than the subject of this letter, from Roche, Merck, AbbVie, Gilead, Pfizer, Vertex, Janssen, Novartis, and Boehringer Ingelheim. The author has received payment for continuing medical education lectures from Roche, Merck, and Gilead. 